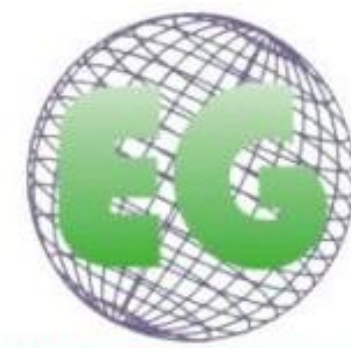

ISSN 1695-6141 $N^{\circ} 56$

\title{
Atención sanitaria trans* competente, situación actual y retos futuros. Revisión de la literatura
}

Competent trans* health care, current situation and future challenges. A Review

\author{
Jesús Manuel García-Acosta, ${ }^{1}$ \\ Maria Elisa de Castro-Peraza ${ }^{2}$ \\ Maria de los Ángeles Arias-Rodríguez ${ }^{3}$ \\ Rosa Llabrés-Solé ${ }^{4}$ \\ Nieves Doria Lorenzo-Rocha ${ }^{4}$ \\ Ana María Perdomo-Hernández ${ }^{4}$
}

(1) Enfermero. Universidad de La Laguna. Servicio Canario de Salud. Tenerife. Islas Canarias. España. jesus.garcia.21@ull.edu.es

(2) PhD. Directora EUE N ${ }^{\underline{a}} \mathrm{~S}^{\underline{a}}$ de La Candelaria. Universidad de la Laguna. Servicio Canario de Salud. Tenerife. Islas Canarias. España.

(3) PhD. Profesora Universidad de La Laguna. Tenerife. Islas Canarias, España.

(4) Profesora EUE N ${ }^{a} S^{\underline{a}}$ de La Candelaria. Universidad de la Laguna. Servicio Canario de Salud. Tenerife. Islas Canarias, España.

\section{http://dx.doi.org/10.6018/eglobal.18.4.357621}

Recibido: 9/01/2019

Aceptado: 20/02/2019

\section{RESUMEN:}

Introducción: Conocemos como una persona trans* a aquella que no se identifica con el sexo de nacimiento, este hecho aún recogido como patología por el actual manual diagnóstico y estadístico de enfermedades mentales (DSM-V) va a provocar que existan desigualdades y barreras significativas a la hora de la atención sanitaria.

Objetivo: Explorar las dificultades para la asistencia sanitaria percibidas por las personas trans* y por los profesionales que los atienden en centros sanitarios.

Material y método: Revisión de la literatura.

Resultados: Tanto los profesionales como las personas trans* perciben barreras significativas. Se podrían agrupar en diversos temas: desigualdades en la atención sanitaria percibidas por los pacientes trans ${ }^{*}$, prejuicios y discriminación sentida, riesgos específicos de salud, déficit de conocimientos de los profesionales y déficit de formación en los planes de estudio actuales. Por otro lado, enfermería ante los pacientes trans* puede resultar un facilitador.

Conclusiones: Existen numerosos factores que pueden ser modificables y que provocan que las personas trans* no perciban una asistencia adecuada. La adecuada formación de los profesionales sanitarios es uno de los que adquiere mayor relevancia Los resultados encontrados aportan una información clave para el futuro diseño de intervenciones dirigidas a mejorar la calidad de la asistencia en este colectivo.

Palabras clave: Personas Transgénero; Educación Continua; Educación en Enfermería; Atención Integral de Salud. 


\section{ABSTRACT:}

Introduction: We know as a transgender person who is not identified with the sex of birth, this fact still collected as pathology by the current Diagnostic and Statistical Manual of Mental Illness (DSM-V) will cause inequalities and barriers At the time of health care.

Objective: To explore the difficulties for health care perceived by trans* people and by the professionals who assist them in health centres.

Material and method: Literature review.

Results: Both professionals and trans ${ }^{*}$ people perceive significant barriers. They could be grouped by diverse topics: healthcare inequalities noticed by trans* patients, prejudices and felt discrimination, specific health risks, lack of professional knowledge and deficit of training in current curricula. On the other hand, nursing with trans* patients may result in a facilitator.

Conclusions: There are multiple factors which can be modified and which produce that trans* people do not notice appropriate care. Appropriate training of health professionals is the one which acquires more relevance. The results found provide key information for the future design of interventions aimed at improving the quality of the assistance in this group.

Keywords: Transgender People; Continuing Education; Education, Nursing; Comprehensive Health Care.

\section{INTRODUCCIÓN}

A las personas antes de llegar a este mundo se les asigna un rol o papel, lo que conocemos como "el deber ser" y que implica castigos por parte de la sociedad en el caso de la no obediencia, y premios para la sujeción al mismo. También ocurre lo mismo con los valores asociados a la feminidad y masculinidad. En función a la genitalidad, se nos impone social y culturalmente un género, y se nos marca un camino a seguir. La heteronormatividad es un fenómeno generalizado en los centros sanitarios y hace que pacientes Lesbianas, Gais, Transexuales, Bisexuales e Intersexuales (LGTBI) sean invisibles, hecho que afecta negativamente al tipo y calidad de la atención que reciben estas personas ${ }^{(1)}$.

El término 'trans' hace referencia a personas con una expresión o identidad de género diferente a la atribuida al nacer ${ }^{(2)}$. Usamos trans* para ser inclusivos de todas las identidades bajo este paraguas, incluyendo transgénero, transexual, género fluido, y otras formas de género "no conforme" ${ }^{(3)}$.

Las personas trans* comparten muchas de las mismas necesidades de salud como la población general, pero pueden tener otras necesidades específicas de la salud, tales como: la terapia hormonal de afirmación de género y la cirugía. Sin embargo, la evidencia sugiere que las personas transexuales a menudo experimentan una mayor tasa de enfermedades, incluyendo los ámbitos de la salud mental, sexual y reproductiva. La exposición a la violencia, la marginación, el estigma y la discriminación también son más altos en esta población (4). Además, experimentan barreras para acceder a la asistencia sanitaria debido al aislamiento, la falta de servicios sociales y personal sanitario competente en el tema ${ }^{(5)}$ y los recursos que determina la salud, tales como la educación, el empleo y la vivienda. Estas barreras son atribuibles en gran medida a la privación jurídica, económica y social, la marginación, la estigmatización y la discriminación, incluyendo el no reconocimiento de su género ${ }^{(4)}$. La evidencia indica que esta transfobia se manifiesta en los centros sanitarios, pero pocos estudios abordan las formas de maltrato experimentado en este contexto ${ }^{(6)}$.

Toda persona tiene derecho a una asistencia sanitaria segura y de calidad. Uno de nuestros papeles como líderes del sector sanitario, es asegurar que futuros profesionales reciban la educación necesaria para proporcionar dichos servicios (7) 
pero en la mayor parte de los casos, el personal sanitario no está familiarizado con la terminología trans* ni diferencian las identidades sexuales ${ }^{(8)}$.

La transexualidad ha sido estudiada desde diferentes perspectivas en el área de investigación ligada a lo clínico con énfasis en lo médico, la psiquiatría, el psicoanálisis y la psicología ${ }^{(9)}$. La investigación que existe relativa a personas trans* y su salud, tienden a tener un punto de vista médico, sobre todo estudios cuantitativos relativos a diferentes aspectos médicos relacionados con la confirmación del género, el acceso y la calidad de la atención ${ }^{(10)}$. Actualmente las minorías sexuales se están convirtiendo en una parte más visible de la población de muchos países, particularmente aquellos en el mundo occidental ${ }^{(11)}$ por lo que se necesita un mayor conocimiento acerca del transgénero, tanto en centros especializados como en centros de atención sanitaria en general. De igual forma un mayor conocimiento en derechos sobre la salud sexual ${ }^{(10)}$.

La Organización Mundial de la Salud (OMS) identifica la falta de investigación y comprensión de la salud LGTBI, junto con actitudes de los proveedores hacia estas poblaciones, como barreras significativas para resolver las disparidades de salud relacionadas con las minorías sexuales ${ }^{(11)}$. Existe un compromiso cada vez mayor en la salud pública de entender y mejorar la salud y el bienestar de las personas transexuales y otras minorías de género, que representan un estimado 0,3-0,5\% (25 millones) de la población mundial ${ }^{(4,12)}$.

La evidencia presentada hasta el momento, demuestra un gran número de aspectos problemáticos de las personas trans ${ }^{*}$ relacionados con la transición y en el acceso a la asistencia sanitaria en general, y éstos tienen efectos negativos sobre la satisfacción de los pacientes ${ }^{(13)}$. La investigación internacional sobre las experiencias de los servicios de salud de las personas LGBTI sugieren que, aunque se han hecho algunos avances, las prácticas discriminatorias continúan existiendo, siendo muchos los pacientes que informan de falta de sensibilidad, prejuicios y prácticas discriminatorias por parte del personal, incluido el personal de enfermería ${ }^{(14)}$.

\section{OBJETIVO}

El objetivo propuesto a través de esta revisión bibliográfica es explorar las dificultades para la asistencia sanitaria percibidas por las personas trans* y por los profesionales que los atienden en centros sanitarios.

\section{METODOLOGÍA}

Se utilizó como diseño una revisión extensa de la literatura de estudios sobre atención sanitaria a pacientes trans* publicados en artículos científicos.

Los artículos de la presente revisión se identificaron a través de la búsqueda automatizada en las siguientes bases de datos científicas: Biblioteca Virtual en Salud (BVS), LILACS, Cuiden, SciELO, PubMed, Scopus, Web of Science (WOS), EBSCOhost y MEDLINE, desde el año 2004 al 2017. Se utilizaron las palabras clave o términos DeCS y MeSH siguientes: "personas transgénero", "educación continua", "educación continua en enfermería", "educación basada en competencias", "educación en salud", "educación en enfermería", "satisfacción del paciente", 
"satisfacción personal", "transgender persons", "continuing education, "continuing nursing education", "health education", "competency-based education", "patient satisfaction" y "satisfaction" recurriendo a los diferentes operadores booleanos ("AND", "OR" y "NOT") junto a los términos relacionados. Se utilizaron varias combinaciones de los términos de búsqueda para ampliar la cobertura de artículos. Se incluyeron estudios cualitativos, cuantitativos y métodos mixtos que cumplieron con los criterios. Adicionalmente se realizó una búsqueda inversa a partir de las referencias bibliográficas de los estudios seleccionados.

El resultado del proceso de búsqueda, con las palabras clave empleadas, permitió seleccionar un total de 57 documentos que cumplieron los criterios de selección. Posteriormente, se procedió a exportar todos los artículos encontrados a la herramienta informática Refworks, con el fin de eliminar duplicados, reduciendo la muestra a 47 documentos. La selección final de los documentos se realizó basándonos en criterios de exclusión: fenómeno no relacionado con el tema a estudio, año de publicación, idioma distinto al español o inglés, relevancia o especificidad y aquellos que estuvieran relacionados con la moralidad o la ética. De esta forma, finalmente quedó una muestra de 34 artículos. Seguidamente tuvo lugar la lectura crítica de los documentos. La validez de los artículos seleccionados fue dada por el grado de evidencias demostrado, por las recomendaciones del artículo y por la aplicabilidad a nuestro contexto.

\section{RESULTADOS}

Todos los artículos incluidos en el estudio son revisiones sin metaanálisis, no existiendo ensayos clínicos aleatorizados. Del total de 34 artículos un 61,76\% (21) recurren a una metodología cualitativa, mientras que un $23,53 \%$ (8) utilizan métodos cuantitativos, siguiendo el restante $14,71 \%$ (5) una metodología mixta cuanticualitativa. La mayor parte de los datos $(53,57 \%)$ se obtuvieron de entrevistas ${ }^{(3,5,7-}$ $10,14-19,33,34)$ y cuestionarios y/o encuestas on-line $(35,71 \%)(6,13,14,20-26)$. Estas metodologías ponen de manifiesto la importancia, según sus autores, de darle voz a los pacientes. El análisis del contenido de los artículos puede consultarse en la tabla 1.

Tabla 1: Análisis del contenido de los artículos seleccionados. Elaboración propia.

\begin{tabular}{|c|c|c|c|c|c|c|c|}
\hline Artículo & Autor/es & Año & País & Metodología & $\begin{array}{l}\text { Cuestionario } \\
\text { pre y post- } \\
\text { intervención }\end{array}$ & Entrevista & $\begin{array}{l}\text { Cuestionario } \\
\text { on-line }\end{array}$ \\
\hline (22) & $\begin{array}{l}\text { Bockting, et } \\
\text { al. }\end{array}$ & 2004 & EE. UU. & Cuantitativa & & & $\checkmark$ \\
\hline (18) & $\begin{array}{l}\text { Bockting, et } \\
\text { al. }\end{array}$ & 2006 & EE. UU. & Cualitativa & & & \\
\hline (25) & $\begin{array}{l}\text { Neville \& } \\
\text { Henrickson. }\end{array}$ & 2006 & $\begin{array}{l}\text { Nueva } \\
\text { Zelanda }\end{array}$ & Cuantitativa & & & $\sqrt{ }$ \\
\hline (26) & $\begin{array}{l}\text { Sanchez et } \\
\text { al. }\end{array}$ & 2006 & EE. UU. & Cuantitativa & & & $\sqrt{ }$ \\
\hline (24) & $\begin{array}{l}\text { Chapman et } \\
\text { al. }\end{array}$ & 2012 & Australia & Mixta & & & $\checkmark$ \\
\hline (27) & $\begin{array}{l}\text { Coleman et } \\
\text { al. }\end{array}$ & 2012 & EE. UU. & Cualitativa & & & \\
\hline (33) & $\begin{array}{l}\text { Snelgrove } \\
\text { et al. }\end{array}$ & 2012 & Canadá & Cualitativa & & & \\
\hline (19) & $\begin{array}{l}\text { Zunner \& } \\
\text { Grace. }\end{array}$ & 2012 & EE. UU. & Cualitativa & & $\checkmark$ & \\
\hline
\end{tabular}




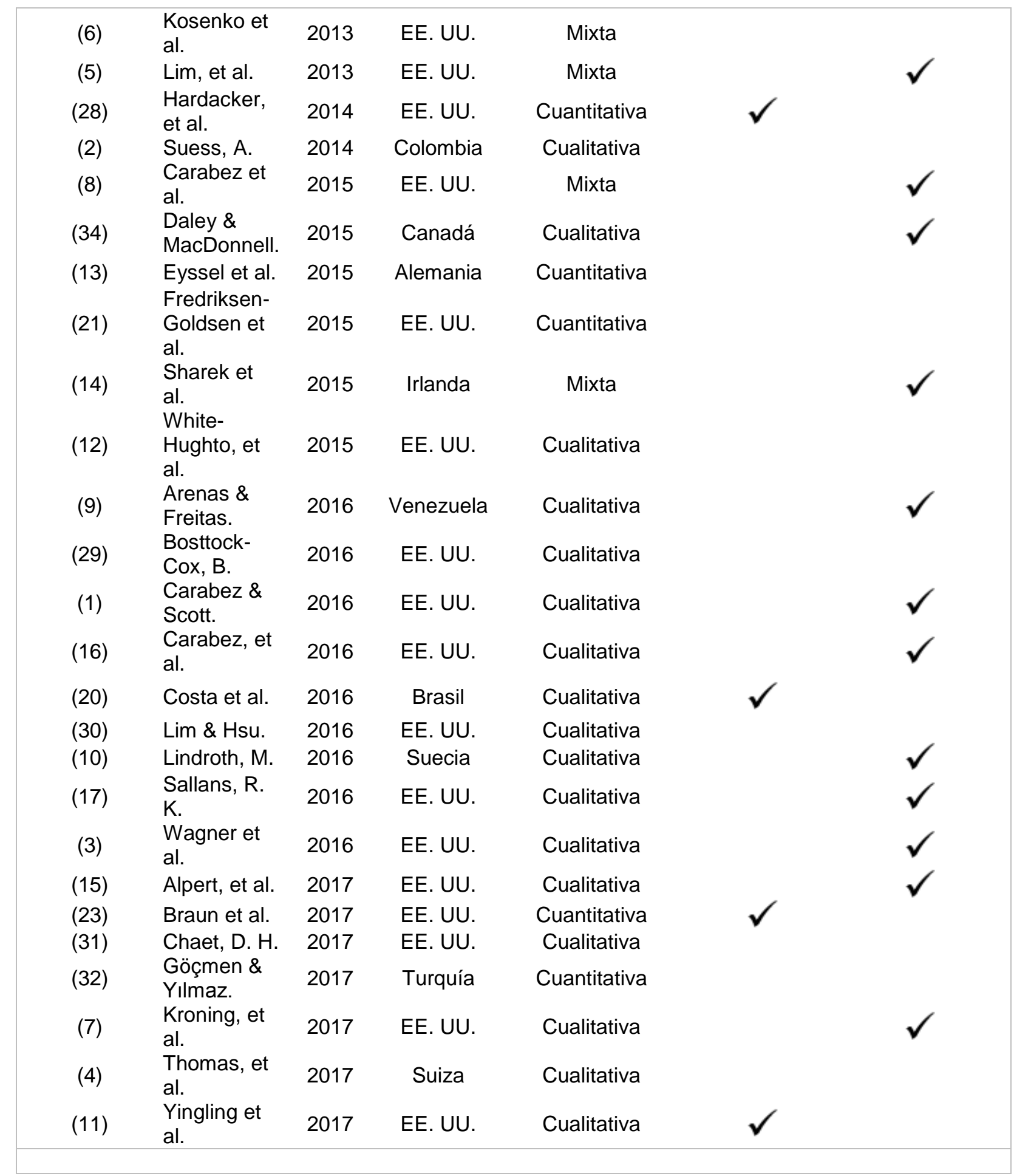

En la figura 1 vemos que la mayor parte de los estudios son estadounidenses $(64,70 \%) \quad(1,3,5-8,11,12,15-19,21-23,26,28-32)$, algunos europeos $(11,76 \%)(4,10,13,14)$, publicaciones sudamericanas $(8,82 \%){ }^{(2,9,20)}$ dos de ellas de habla hispana ${ }^{(2,9)}$ y de otros paises $(14,70 \%)$ como Turquía ${ }^{(32)}$, Australia ${ }^{(24)}$, Nueva Zelanda ${ }^{(25)}$ y Canadá $(33,34)$. No hemos encontrado ningún estudio asiático así como tampoco se ha encontrado ningún estudio español, que relacione la falta de conocimientos de los profesionales sanitarios con la satisfacción percibida por los pacientes trans*. 
Figura 1: Análisis del porcentaje de publicación y año de publicación por áreas geográficas. Elaboración propia

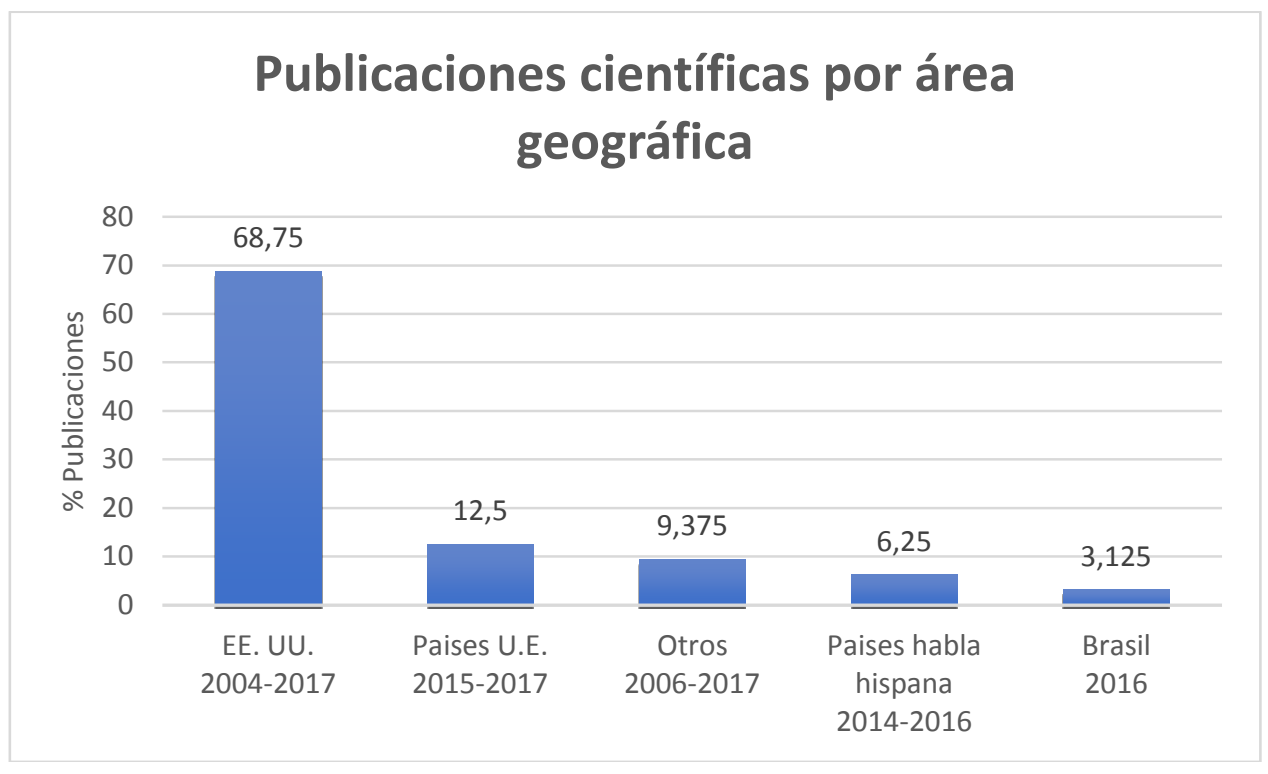

De la literatura consultada, cuatro son los estudios que proponen acciones formativas $(11,20,23,28)$, uno de ellos reporta el doble de efecto positivo en mujeres respecto a hombres ${ }^{(20)}$ y otros tres los que estudian la salud de personas mayores LGTBI $(14,21,28)$

\section{DISCUSIÓN}

Los resultados de esta revisión muestran que tanto los profesionales como las personas trans ${ }^{*}$ perciben barreras significativas. Se podrían agrupar en diversos temas (Tabla 2).

Tabla 2: Barreras a la atención sanitaria. Elaboración propia.

\begin{tabular}{|c|c|c|}
\hline Barrera & Estudios & Síntesis principal \\
\hline $\begin{array}{l}\text { Desigualdades en la atención } \\
\text { sanitaria percibidas por los } \\
\text { pacientes trans*. }\end{array}$ & $\begin{array}{c}10,11,13,14, \\
16, \\
21,22,24,26, \\
27,28,30,33\end{array}$ & $\begin{array}{l}\text { Existe desinterés de los profesionales } \\
\text { por la formación y las necesidades del } \\
\text { colectivo. Existe falta de investigación } \\
\text { respecto al envejecimiento, actitudes de } \\
\text { los estudiantes, la transexualidad en la } \\
\text { infancia y la salud reproductiva. La } \\
\text { investigación existente se ha centrado en } \\
\text { la satisfacción de resultados quirúrgicos } \\
\text { y no en la satisfacción como paciente. La } \\
\text { experiencia en la asistencia recibida no } \\
\text { es satisfactoria. }\end{array}$ \\
\hline $\begin{array}{c}\text { Prejuicios y discriminación } \\
\text { sentida. }\end{array}$ & $\begin{array}{c}2,6,7,8,10, \\
13, \\
14,15,16,17, \\
19, \\
20,21,22,23, \\
24, \\
25,26,29,30, \\
31,32\end{array}$ & $\begin{array}{l}\text { Los pacientes manifiestan haber sentido } \\
\text { discriminación en forma de: acoso } \\
\text { verbal, acoso físico, desprecio, actitudes } \\
\text { negativas y negación de los cuidados, } \\
\text { además de la presunción de } \\
\text { heterosexualidad. Los profesionales no } \\
\text { son capaces de identificar la } \\
\text { discriminación. Los estudios que valoran } \\
\text { los niveles de prejuicios indican que los } \\
\text { niveles más altos los encontramos entre }\end{array}$ \\
\hline
\end{tabular}




\begin{tabular}{|c|c|c|}
\hline & & $\begin{array}{l}\text { varones, religiosos conservadores y sin } \\
\text { educación. }\end{array}$ \\
\hline Riesgos específicos de salud. & $\begin{array}{c}3,5,6,7,11 \\
16, \\
18,21,25,27 \\
29,32 \\
\end{array}$ & $\begin{array}{l}\text { El riesgo de salud en este colectivo está } \\
\text { incrementado tanto en trastornos físicos } \\
\text { como en trastornos psicológicos y } \\
\text { sociales. }\end{array}$ \\
\hline $\begin{array}{l}\text { Déficit de conocimientos de los } \\
\text { profesionales. }\end{array}$ & $\begin{array}{c}1, \\
7,10,11,14, \\
15,17,22,23, \\
28,33,34\end{array}$ & $\begin{array}{l}\text { Los profesionales sanitarios en ningún } \\
\text { momento de su formación adquieren } \\
\text { conocimientos sobre la atención a } \\
\text { pacientes trans }{ }^{*} \text { y desconocen los } \\
\text { recursos para obtener información, lo } \\
\text { que conlleva a una desigualdad en los } \\
\text { cuidados prestados. }\end{array}$ \\
\hline $\begin{array}{l}\text { Déficit de formación en los planes } \\
\text { de estudio actuales. }\end{array}$ & $\begin{array}{l}1,5,8,11,14 \\
15,25,27,29\end{array}$ & $\begin{array}{l}\text { Los planes de estudio deben ser } \\
\text { revisados, pues no proporcionan una } \\
\text { preparación suficiente, existiendo una } \\
\text { necesidad crítica en educación de } \\
\text { identidad de género, en terminología } \\
\text { relacionada con la orientación sexual y } \\
\text { en necesidades y sexualidad de las } \\
\text { personas mayores, con especial énfasis } \\
\text { en las personas trans. Un plan de } \\
\text { estudios que promueva la diversidad } \\
\text { sexual en la educación posteriormente } \\
\text { mejorará la atención sanitaria. Se } \\
\text { necesita específicamente hacer frente a } \\
\text { la heteronormatividad y a la homofobia y } \\
\text { transfobia dentro de los entornos de } \\
\text { asistencia sanitaria. }\end{array}$ \\
\hline
\end{tabular}

\section{Desigualdades en la atención sanitaria percibidas por los pacientes trans*}

Según los testimonios de las personas trans*, los profesionales no empatizan con ellos, mostrando cierto desinterés por conocer y entender las necesidades que su colectivo de personas requiere. Así, encuentran como no satisfactoria la asistencia recibida ${ }^{(33)}$.

No existe suficiente investigación centrada en las personas trans* y sus experiencias en salud sexual, fundamentalmente de enfoque cualitativo ${ }^{(10)}$ pese a que resulta crucial investigar lo que las personas trans* sienten, sus necesidades y miedos en materia de asistencia sanitaria sobre todo con respecto a la transición ${ }^{(11,13,16)}$.

En el pasado, los estudios de investigación se han centrado en la satisfacción de los pacientes con respecto a los resultados de cirugía, siendo pocos los estudios que han abordado la satisfacción del paciente respecto al proceso y servicios ofrecidos ${ }^{(13)}$, pese a que las encuestas de satisfacción han llegado a ser ampliamente aceptadas como una herramienta importante en la planificación de la mejora de la calidad ${ }^{(22)}$.

Con respecto al envejecimiento con éxito y cuidado de personas mayores LGTBI, pocos son los hallazgos en la literatura existente ${ }^{(14,21,28)}$ al igual que ocurre con los estudios que evalúan las actitudes de los estudiantes de ciencias de la salud frente a personas LGTBI ${ }^{(24,26,30)}$ y los que tratan la disforia de género en edad temprana y relacionados con la salud reproductiva ${ }^{(27)}$. Las investigaciones sobre las experiencias de los servicios de salud de las personas LGTBI sugieren que las prácticas 
discriminatorias por parte del personal continúan existiendo, manifestando que existe falta de sensibilidad y prejuicios ${ }^{(14)}$.

\section{Prejuicios y discriminación sentida}

Existen estudios que valoran los niveles de prejuicios hacia personas LGTBI, siendo los niveles más altos entre personas religiosas conservadoras ${ }^{(20,24)}$, sin educación y de sexo masculino ${ }^{(20,30)}$. Este prejuicio afecta también a los profesionales de la salud, entre los estudiantes de enfermería el prejuicio es frecuente, explícito y creciente ${ }^{(20)}$, una cuarta parte del personal médico encuestado tiene una opinión negativa acerca de los grupos LGTBI ${ }^{(32)}$ les genera malestar y desconfianza ${ }^{(6)}$, incluso los profesionales mostraron abiertamente preferencia en el trato a heterosexuales y hombres y mujeres homosexuales, frente a personas trans* ${ }^{*}$.

La presunción de heterosexualidad supone uno de los obstáculos más importantes a la hora de recibir y proporcionar atención sanitaria de calidad a las personas LGTBI ${ }^{(24,25)}$. Entre los estereotipos más comunes a los que se enfrentan las personas LGTBI se encuentran: la relación entre homosexual, persona transexual y el virus de la inmunodeficiencia humana $(\mathrm{VIH})$, además de la hipótesis de que las mujeres homosexuales no presentan riesgos de padecer enfermedades de transmisión sexual $(\mathrm{ETS})^{(15)}$.

Los profesionales no tienen conocimientos básicos para identificar la discriminación, incluso la perpetuada por ellos mismos ${ }^{(20)}$. La discriminación es percibida tanto de forma directa como indirecta ${ }^{(32)}$ y en el entorno de las enfermeras ${ }^{(10)}$. Cuando se les pregunta a los pacientes acerca de la discriminación, más de la mitad dice haberse sentido discriminado en centros sociosanitarios ${ }^{(10,14)}$, un $14 \%$ en centros de atención sanitaria $^{(31,32)}, 28 \%$ había sufrido acoso verbal ${ }^{(2,13)}$ y el $2 \%$ acoso físico ${ }^{(13)}$. Una cuarta parte de los pacientes LGTBI perciben que son tratados de forma distinta, con desprecio $^{(19)}$ y notaron un cambio de actitud hacia una actitud negativa al revelar su orientación o identidad sexual ${ }^{(15)}$, incluso al $19 \%$ se le había negado el cuidado $(2,13,16)$. Además, una quinta parte informó de que sus preferencias estaban excluidas respecto a las decisiones médicas ${ }^{(15)}$, siendo también el acceso a la asistencia sanitaria difícil en varios niveles ${ }^{(13,21,23)}$.

Al preguntar a los profesionales de la salud, un 5\% utilizaba las formas inclusivas de género, el $44 \%$ no conocía las formas inclusivas, el $37 \%$ no sabía a qué se refería el género y el $14 \%$ confundía la identidad de género con la orientación sexual ${ }^{(8)}$. Investigaciones recientes indican que las actitudes negativas hacia las minorías sexuales persisten entre los profesionales de enfermería ${ }^{(30)}$, revelando que se sienten incómodos al tratar a personas LGTBI ${ }^{(25)}$.

Muchos pacientes evitan mencionar su orientación sexual o la sexualidad en general $(10,24)$, incluso si la información puede resultar útil para el personal sanitario ${ }^{(32)}$, por temor a una respuesta negativa ${ }^{(14)}$ o una experiencia negativa anterior ${ }^{(17,22,23,25,26,29)}$. En el caso de personas trans*, hasta el 30\% evita o retrasa la búsqueda de los servicios de salud ${ }^{(17,30)}$, sufren aprehensión, temor al acoso o discriminación ${ }^{(3)}$, y recurren a tratamientos hormonales ilegales a través de internet ${ }^{(29)}$ pese a que pueda tener efectos perjudiciales sobre su salud ${ }^{(7)}$. Con respecto a mujeres, son estas las que divulgan con mayor frecuencia su orientación e identidad sexual ${ }^{(25)}$. 


\section{Riesgos específicos de salud}

Dentro de la población LGTBI existe un mayor riesgo de sufrir trastornos del estado de ánimo, contraer el VIH ${ }^{(3,5,7,16,25)}$, sobrepeso, obesidad ${ }^{(5,21)}$, ETS ${ }^{(3,5,7)}$ y problemas de salud mental ${ }^{(5,29)}$ como ansiedad ${ }^{(5)}$ y depresión ${ }^{(18,21)}$. Además, presentan mayor consumo de tabaco, alcohol y drogas ${ }^{(7,11,16,29)}$, de 2 a 3 veces más probabilidad de intento de suicidio ${ }^{(2,5,16,18)}$, estigmatización ${ }^{(3,5,6,16,29)}$ que conduce a prejuicios y discriminación ${ }^{(27)}$, y falta de apoyo social ${ }^{(18,21)}$. Una encuesta on-line acerca de la discriminación, informó que un $43,2 \%$ de los encuestados habían intentado suicidarse al menos una vez en su vida ${ }^{(32)}$.

\section{Déficit de conocimientos de los profesionales}

Los profesionales asistenciales, en ningún momento de su formación académica, han adquirido conocimientos acerca de la atención a pacientes trans ${ }^{*}$ (ni se ve reflejado en los planes de estudios).

Además de no tener conocimientos teóricos sobre el tema, tampoco conocen los recursos de los que disponen para obtener información. Esto provoca que a la hora de la asistencia se desconozcan los procedimientos para ayudarles y den como resultado situaciones difíciles por falta de familiaridad con la terminología tratada ${ }^{(33,34)}$.

Mejorar la salud de las personas LGTBI requiere de un personal competente en la atención ${ }^{(15)}$, se necesita un mayor conocimiento acerca del transgénero tanto en centros de atención especializada como de atención sanitaria en general ${ }^{(10,14)}$, casi el $50 \%$ de los profesionales informantes clave informó de falta de conocimiento de necesidades y consideraciones especiales frente a personas LGTBI ${ }^{(1,22,23)}$. Esta competencia a adquirir por los profesionales de salud se debe centrar sobre todo en lo relacionado a la conducta y el lenguaje verbal, de forma que se mejore la confianza con el paciente ${ }^{(17)}$ permitiendo llevar a cabo una atención eficaz y accesible ${ }^{(7,15)}$, reduciendo las barreras en la atención ${ }^{(7)}$, mejorando los resultados de la salud para los profesionales que atienden ${ }^{(28)}$ y proporcionando un servicio inclusivo y sensible $(11,14)$. Hay muchas oportunidades para aumentar el conocimiento sobre la salud transgénero ${ }^{(17)}$. Al consultar a los pacientes, solo uno de cada tres creyó que los profesionales de salud tenían suficientes conocimientos sobre temas LGTBI ${ }^{(14)}$.

\section{Déficit de formación en los planes de estudio actuales}

La universidad debe proporcionar a los estudiantes de ciencias de la salud, la oportunidad de organizar y ser parte activa de grupos de interés, de forma que se cultiven habilidades de liderazgo y sirvan de estímulo y apoyo a la formación de otros grupos de interés ${ }^{(5)}$. Los planes de estudio de enfermería, y en general de ciencias de la salud, deben ser revisados respecto a los temas relacionados con LGTBI ${ }^{(5)}$, pues no proporcionan una preparación suficiente ${ }^{(1)}$, existiendo una necesidad crítica en educación de identidad de género, en terminología relacionada con la orientación sexual ${ }^{(8)}$ y en necesidades y sexualidad de las personas mayores, con especial énfasis en las personas trans ${ }^{\star}{ }^{(14)}$. Un plan de estudios que promueva la diversidad sexual en la educación posteriormente mejorará la atención sanitaria de personas LGTBI ${ }^{(5)}$. El reto para la educación en enfermería es sintetizar la evidencia creciente de los problemas de salud de LGTBI ${ }^{(30)}$, se necesita específicamente hacer frente a la heteronormatividad y a la homofobia y transfobia dentro de los entornos de asistencia sanitaria ${ }^{(25)}$. 
Los estudiantes deben ser alentados a ir más allá de la evaluación de las manifestaciones físicas o indicadores de salud ${ }^{(5)}$. Los estudiantes a los que se les ha encuestado manifiestan haber sentido ansiedad por no tener la preparación suficiente para prestar un adecuado cuidado en sus prácticas clínicas (11). Los resultados de programas educativos mostraron que los estudiantes se sintieron cómodos con la formación en temas relacionados con LGTBI ${ }^{(15)}$, y que lo aprendido les es relevante para su práctica clínica ${ }^{(11)}$. Al estudiar qué ocurría dentro de las escuelas de enfermería, cuatro de cada diez estudiantes trans* de enfermería sintieron discriminación debido a su identidad de género dentro de la propia escuela de enfermería ${ }^{(32)}$, esta transfobia puede tener un impacto duradero en su práctica profesional $^{(30)}$.

Los docentes de ciencias de la salud deben proporcionar diversas prácticas clínicas, grupos de interés y expectativas claras para elaborar unos planes de cuidado sensibles con respecto a pacientes LGTBI, pudiendo utilizar su relación con los estudiantes como una forma de discusión y fomento de perspectivas positivas. Además deben asegurar el fomento del desarrollo de conocimientos capaces de implementar mejores prácticas en el cuidado de pacientes LGTBI $^{(5)}$.

\section{Enfermería como facilitador ante los pacientes trans*}

Los profesionales de enfermería son de particular interés, puesto que son cuidadores directos básicos ${ }^{(5)}$, están dotados de poder de decisión ${ }^{(13)}$, tiene el compromiso de proporcionar una atención integral ${ }^{(25)}$ y un papel importante que desempeñar en la lucha por la equidad y justicia dentro de la atención sanitaria (10), y en la eliminación de las disparidades con respecto a la salud de las personas LGTBI ${ }^{(5,7)}$. Este papel cobra especial importancia en las personas trans*, desde las primeras conversaciones que puedan tener acerca de sus sentimientos a través de la reasignación de sexo, hasta el apoyo y seguimiento durante toda su vida sobre sus condiciones de salud físicas y mentales ${ }^{(29)}$. La mayoría de las enfermeras no están preparadas, carecen de conocimientos ${ }^{(1,11,16)}$ y no están familiarizadas con la terminología trans* ni diferencian las identidades sexuales. Este desconocimiento puede, de forma inadvertida, excluir a pacientes y su familia haciéndoles invisibles evitando que se le preste un cuidado adecuado ${ }^{(8)}$. Las intervenciones dirigidas a los miembros de la familia resultan tener efectos positivos, al fomentar la comprensión y la aceptación de las personas trans* por sus seres queridos ${ }^{(12)}$. Con respecto a las personas mayores LGTBI, las enfermeras deben de ser conscientes del riesgo de aislamiento y soledad que este grupo presenta ${ }^{(14)}$.

Las investigaciones con enfoque participativo ${ }^{(10,13,20,28)}$ se emplean cada vez más en la investigación en ciencias de la salud ${ }^{(13)}$. Dada la formación y el papel que enfermería representa para el paciente, este tipo de investigación es de gran aplicabilidad.

\section{CONCLUSIONES}

Esta revisión presenta una serie de barreras identificadas por la comunidad trans* y por los propios profesionales de la salud, que limitan el acceso a los cuidados y la calidad de estos. Asimismo, tanto pacientes como profesionales refieren una formación sanitaria inadecuada, lo que lleva a un déficit en la prestación de los servicios de salud. 
A pesar de que existen barreras significativas, este estudio vislumbra la existencia de una serie de prácticas que pueden representar soluciones potenciales. Estas incluyen mejorar las fuentes de información (guías de práctica clínica, folletos divulgativos, algoritmos de atención sanitaria), mejorar la diseminación de la información que ya existe, incorporar formación sobre salud trans* a los actuales currículos profesionales, incluir formación específica en los actuales planes de estudio de los futuros profesionales, mejorar la colaboración entre profesionales más expertos e iniciativas basadas en líneas políticas para mejorar el acceso a los servicios de salud de los pacientes trans.

Sin embargo, los profesionales de la salud poseen un espectro de actitudes y creencias que impactan en la atención que reciben estos pacientes. Muchas inadecuaciones en el cuidado pueden ser debidas a una cultura de heterosexualidad. Es precisa más investigación sobre intervenciones que puedan facilitar la perdida de barreras ante la revelación de la orientación sexual y más aún del sexo sentido buscando romper las asunciones de heteronormatividad por parte del equipo de salud.

\section{REFERENCIAS}

1. Carabez R, \& Scott M. "Nurses don"t deal with these issues': nurses' role in advance care planning for lesbian, gay, bisexual and transgender patients. J Clin Nurs. 2016;25(23-24):3707-15.

2. Suess A. Cuestionamiento de dinámicas de patologización y exclusión discursiva desde perspectivas trans e intersex. Rev Estud Soc. 2014;9(49):12843.

3. Wagner PE, Kunkel A, Asbury MB, Soto F. Health (Trans) gressions: Identity and Stigma Management in Trans* Healthcare Support Seeking. Women Lang. 2016;39(1):49-74. 26p.

4. Thomas, R., Pega, F., Khosla, R., Verster, A., Hana, T., \& Say L. Ensuring an inclusive global health agenda for transgender people. Bull World Heal Organ [Internet]. 2017;95(August 2016):154-6. Available from: http://dx.doi.org/10.2471/BLT.16.183913

5. Lim FA, Brown D V., Jones H. Lesbian, Gay, Bisexual, and Transgender Health: Fundamentals for Nursing Education. J Nurs Educ [Internet]. 2013;52(4):198203. Available from: http://www.healio.com/doiresolver?doi=10.3928/0148483420130311-02

6. Kosenko K, Rintamaki L, Raney S, Maness K. Transgender Patient Perceptions of Stigma in Health Care Contexts. Med Care [Internet]. 2013;51(9):819-22. Available from: http://content. wkhealth.com/linkback/openurl?sid=WKPTLP:landingpage \&an $=00$ 005650-201309000-00010

7. Kroning, M., Green, J., \& Kroning K. Dimensions of inclusive care. A young transgender patient sparks the need for an immediate education action plan. Nurs Manage. 2017;48(1):1.

8. Carabez R, Pellegrini M, Mankovitz A, Eliason M, Scott M. Does your organization use gender inclusive forms? Nurses' confusion about trans* terminology. J Clin Nurs. 2015;24(21-22):3306-17.

9. Arenas $Y, \&$ Freitas M.G. El contexto social y la comprensión psicoterapéutica en la transexualidad. TRILOGÍA Ciencia, Tecnol y Soc. 2016;8:11-25.

10. Lindroth M. "Competent persons who can treat you with competence, as simple 
as that"- An interview study with transgender people on their experiences of meeting health care professionals. J Clin Nurs. 2016;25(23-24):3511-21.

11. Yingling CT, Cotler K, Hughes TL. Building nurses' capacity to address health inequities: incorporating lesbian, gay, bisexual and transgender health content in a family nurse practitioner programme. J Clin Nurs. 2017;26(17-18):2807-17.

12. White Hughto JM, Reisner SL, Pachankis JE. Transgender stigma and health: A critical review of stigma determinants, mechanisms, and interventions. Soc Sci Med [Internet]. 2015;147:222-31. Available from: http://dx.doi.org/10.1016/j.socscimed.2015.11.010

13. Eyssel J, Koehler A, Dekker A, Sehner S, Nieder TO. Needs and concerns of transgender individuals regarding interdisciplinary transgender healthcare: $A$ non-clinical online survey. PLoS One. 2017;12(8).

14. Sharek DB, Mccann E, Sheerin F, Glacken M, Higgins A. Older LGBT people's experiences and concerns with healthcare professionals and services in Ireland. Int J Older People Nurs. 2015;10(3):230-40.

15. Alpert AB, CichoskiKelly EM, Fox AD. What Lesbian, Gay, Bisexual, Transgender, Queer, and Intersex Patients Say Doctors Should Know and Do: A Qualitative Study. J Homosex. 2017;64(10):1368-89.

16. Carabez, R. M., Eliason, M. J., \& Martinson M. Nurses' Knowledge About Transgender Patient Care: A Qualitative Study. Adv Nurs Sci [Internet]. 2016;39(3):257-71. Available from: http://content.wkhealth.com/linkback/openurl?sid=WKPTLP:landingpage\&an=00 012272-201607000-00007

17. Sallans RK. Lessons from a Transgender Patient for Health Care Professionals. AMA J ethics [Internet]. 2016;18(11):1139-46. Available from: http://journalofethics.ama-assn.org/2016/11/mnar1-

1611.html\%0Ahttp://www.ncbi.nlm.nih.gov/pubmed/27883306

18. Bockting, W. O., Knudson, G., \& Goldberg JM. Counseling and Mental Health Care for Transgender Adults and Loved Ones. Int J Transgenderism. 2006;9(34):83-94.

19. Zunner BP, Grace PJ. The Ethical Nursing Care of Transgender Patients. AJN, Am J Nurs [Internet]. 2012;112(12):61-4. Available from: http://content.wkhealth.com/linkback/openurl?sid=WKPTLP:landingpage\&an=00 000446-201212000-00034

20. Costa AB, Pase PF, de Camargo ES, Guaranha C, Caetano AH, Kveller D, et al. Effectiveness of a multidimensional web-based intervention program to change Brazilian health practitioners' attitudes toward the lesbian, gay, bisexual and transgender population. J Health Psychol [Internet]. 2016;21(3):356-68. Available from: http://journals.sagepub.com/doi/10.1177/1359105316628748

21. Fredriksen-Goldsen KI, Kim H-J, Shiu C, Goldsen J, Emlet CA. Successful Aging Among LGBT Older Adults: Physical and Mental Health-Related Quality of Life by Age Group. Gerontologist [Internet]. 2015;55(1):154-68. Available from: https://academic.oup.com/gerontologist/gerontologist/article/2957461/Successful

22. Bockting W, Robinson B, Benner A, Scheltema K. Patient satisfaction with transgender health services. J Sex Marital Ther. 2004;30(4):277-94.

23. Braun HM, Ramirez D, Zahner GJ, Gillis-Buck EM, Sheriff H, Ferrone M. The LGBTQI health forum: An innovative interprofessional initiative to support curriculum reform. Med Educ Online [Internet]. 2017;22(1). Available from: http://dx.doi.org/10.1080/10872981.2017.1306419

24. Chapman R, Watkins R, Zappia T, Nicol P, Shields L. Nursing and medical students' attitude, knowledge and beliefs regarding lesbian, gay, bisexual and transgender parents seeking health care for their children. J Clin Nurs. 
2012;21(7-8):938-45.

25. Neville S, Henrickson M. Perceptions of lesbian, gay and bisexual people of primary healthcare services. J Adv Nurs. 2006;55(4):407-15.

26. Sanchez NF, Rabatin J, Sanchez JP, Hubbard S, Kalet A. Medical students' ability to care for lesbian, gay, bisexual, and transgendered patients. Fam Med. 2006;38(1):21-7.

27. Coleman E, Bockting W, Botzer M, Cohen-Kettenis P, DeCuypere G, Feldman J, et al. Standards of Care for the Health of Transsexual, Transgender, and Gender-Nonconforming People, Version 7. Int J Transgenderism. 2012;13(4):165-232.

28. Hardacker CT, Rubinstein B, Hotton A, Houlberg M. Adding silver to the rainbow: The development of the nurses' health education about LGBT elders (HEALE) cultural competency curriculum. J Nurs Manag. 2014;22(2):257-66.

29. Bosttock-Cox B. The role of primary care nurses in the care of transgender people. Pract Nurse. 2016;46(9):26-31.

30. Lim, F. A., \& Hsu R. Nursing Students' Attitudes Toward Lesbian, Gay, Bisexual, and Transgender Persons: An Integrative Review. Nurs Educ Perspect [Internet]. 2016;37(3):144-52. Available from: https://libproxy.singaporetech.edu.sg/login?url=http://search.ebscohost.com/logi n. aspx?direct=true\&AuthType $=$ cookie, ip, $u$ id $\& d b=c 8 h \& A N=115441563 \&$ site $=e d s-$ live

31. Chaet DH. The AMA Code of Medical Ethics' Opinions Related to Discrimination and Disparities in Health Care. AMA J Ethics April. 2017;19(1):54-62.

32. Göçmen I, Yılmaz V. Exploring Perceived Discrimination Among LGBT Individuals in Turkey in Education, Employment, and Health Care: Results of an Online Survey. J Homosex. 2017;64(8):1052-68.

33. Snelgrove, J. W., Jasudavisius, A. M., Rowe, B. W., Head, E. M., \& Bauer GR. "completely out-at-sea" with "two-gender medicine": A qualitative analysis of physician-side barriers to providing healthcare for transgender patients. BMC Health Serv Res. 2012;12(1).

34. Daley, A., \& MacDonnell JA. "That would have been beneficial": LGBTQ education for home-care service providers. Heal Soc Care Community. 2015;23(3):282-91. 